\title{
Pneumatization patterns of human sphenoid sinus associated with the internal carotid artery and optic nerve by CT scan
}

\author{
Mohammad Ahmad Abdalla \\ Department of Anatomy, Tikrit University College of Medicine, Tikrit, Iraq
}

\begin{abstract}
Introduction. The sphenoid sinus is an unique space arises within the nasal capsule of the embryonic nose and remains undeveloped until age three years. It appears before birth in small size; but a recognized definitive cavity revealed at birth. Secondary pneumatization does not begin until ten years old and finally by age 18 it reaching the full size.

Aim. To demonstrate the extent of sphenoid sinus pneumatization and identify the extent of pneumatization for two related anatomical structures, the internal carotid artery and optic nerve by CT scanning.

Materials and methods. A cross-sectional study with randomized sample of 250 individuals attending the Radiology Section of both Baghdad Medical City and Salah Aldeen Teaching Hospitals from February 2019 to January 2020. All the participated adult individuals were undergo examination for the extent of sphenoid sinus pneumatization with two anatomically neighbor structures, the internal carotid artery and optic nerve, by CT scanning.

Outcomes. Three patterns of sphenoid sinus pneumatization were recognized, the pre-sellar, sellar and post-sellar with prevalence $11.2 \%, 14 \%$ and $74.8 \%$ respectively. The internal carotid artery was pneumatized within sphenoid sinus in four differentiated types with prevalence $10 \%, 30.8 \%, 56 \%$ and $3.2 \%$ respectively; while the optic nerve was pneumatized within the same sinus in five distinguishable types with prevalence $4 \%, 52 \%, 34 \%, 3.6 \%$ and $6.4 \%$ respectively had been reported.

Conclusions. A higher prevalence of optic nerve extension into the lateral wall of sphenoid sinus causing bulged optic canal in same sinus, also superio-posterior aspect of it tends to be more pneumatized than other aspects; all these findings can be with magnificent importance to determine the gender, age or even race and ethnic group of alive or dead individuals.
\end{abstract}

Keywords: computed tomography (CT), pneumatization, sphenoid paranasal sinus, internal carotid artery, optic nerve

\section{INTRODUCTION}

The sphenoid paranasal sinus is situated mainly inside the sphenoid bone as a twin structure. The cavities or chambers of these paired sinuses are divided by a specific bony septum, situated in the mid-sagittal plane; some asymmetrical features in their shape and size are almost always found, but great septal deviations are slightly rare (1). This sinus is an unique space that it do not arises from outpouchings of the nasal cavity, but within the nasal capsule of the embryonic nose and remains undeveloped until age three years (2). It begins to develop during third fetal month when the sphe- no-ethmoidal recesses constrict with the adjacent superior conchae draw upward. It appears before birth with small size; but a recognized definitive cavity revealed at birth, while after five years, it invades the presphenoid bone and there are other successive periods of rapid growth. Secondary pneumatization does not begin until ten years old and finally by age 18 , the sinus reaching its full size $(3,4)$.

Many important anatomical structures are surrounding the sphenoid sinus as it situated in the center of the skull; such as superiorly to it are the olfactory tracts, pituitary gland, frontal lobes of 
forebrain, with a wide-spread intercavernous venous plexus. While the optic chiasma is located antero-superiorly; but in anterior position, the anterior wall of sphenoid bone configures a small part in the posterior margin of the orbit; inferiorly, in addition to the nasopharynx, the nerve and the blood vessels related to the pterygoid canal, that present antero-posteriorly immediately under the sphenoid sinus floor (5-7).

The sphenoid sinus walls are usually irregular bony septa, which partially divide the recesses forming incomplete compartments in that sinus. Characteristic dehiscences may be found within that bony wall of the sinus, more obvious superiorly and laterally. Those dehiscences can cause direct connections between the sphenoid sinus mucosa with overlying dura mater. A thin bony border with incidental dehiscences isolates sphenoid sinus laterally from the internal carotid artery and cavernous sinus, while the maxillary branch of trigeminal cranial nerve is found alongside the inferior margin of sphenoid sinus $(8,9)$.

The optic nerve with internal carotid artery are the only two adjacent structures to the sphenoid sinus that may possibly emerge into its cavity in some cases that increasing their susceptibility for injury during specific surgical procedures like endoscopic intranasal operation (10). The ostium of sphenoid sinus is located high on anterior sinus wall approximately one-third to one-half above the front of the sinus. Sphenoid sinus is draining into the posterior part of the sphenoethmoidal recess, over the superior turbinate level (11).

Computed tomography (CT) is a specific medical technique, which yields images in trans-axial plane across the individual body. Any single CT image is usually reconstructed in mathematical methods from a massive number of definitive one-dimensional screenings of the particular plane. Those screenings are obtained electronically utilizing a linear order of solid-state sensors with an $\mathrm{x}$-ray source, which turn around the individual (12). The highly resolute CT sections supplies adorable bone details with delicate soft tissues mapping. It is a standard procedure used prior to most of endoscopic surgeries to assess the extension of the inflammation area and to determine the important anatomical landmarks with their recognizable variations (13).

CT and to a lesser degree, MRI, supplies the radiologists and otolaryngologists with wonderful images for the ostiomeatal region with another nasal and paranasal anatomical structures (14-16). Therefore, it considers as the most precise technique utilized for examination of many lesions present in any surface or part of the paranasal sinus, permitting an excellent access to each wall of it and can be used as there is a clear-cut clue of paranasal sinus pathology (17). Furthermore, it used to assess the sinonasal cavity and gives a proper evaluation for the paranasal sinus structures, craniofacial bone details and the extension of pneumatization for each paranasal sinus (18).

This study aims to demonstrate the extent of sphenoid sinus pneumatization and identify the extent of pneumatization for two related anatomical structures, the internal carotid artery and optic nerve by axial, sagittal and coronal $\mathrm{CT}$ sections scanning.

\section{MATERIALS AND METHODS}

A cross-sectional study of randomized sample of two hundreds-fifty cases who were apparently healthy normal subjects attending the Radiology Section of both Baghdad Medical City and Salah Aldeen Teaching Hospitals in Iraq during the period from February 2019 to January 2020.

The current study was approved by the Medical Ethics Committee of Tikrit University College of Medicine (Code IQ.TUCOM.REC.19.4692). Ethical approval statements were acquired for all participated individuals, depending upon Helsinki Declaration of World Medical Association; with it last revision at Edinburgh in 2000.

This study included individuals who had complained of headache and referred for radiology section to have CT scans for brain and paranasal sinuses in which no pathological findings were detected in these sinuses. On the other hand, patients with history of surgical interference, trauma, or any pathological lesion at the skull or maxillofacial area were excluded from the present study.

Sections of five-millimeters were often quite enough to evaluate various sinonasal or basal skull structures, but thinner sections of three-millimeters were used for identifying small structures and evaluating the entire ostiomeatal unit. In terms of filming, the recommend intermediate window width/level (W/L) technique was (2500/250, W/L) (19). The imaging protocols used in the current 
study including: 3 to $5 \mathrm{~mm}$ slice thickness, 140 $\mathrm{kVp}, 94 \mathrm{mAs}, 2000$ window width, 400 window level and 35-45 second scan time.

Using 3D program of the CT Siemens workstation with a resolution max. $1280 \times 1042$ full screen formats and a picture size $360 \mathrm{~mm}$ x $288 \mathrm{~mm}$. The workstation permitted synchronous viewing of particular reference point at three view sections (the axial, sagittal and coronal); then capture these images with JPEG format and downloaded on CD for transfer its data. Specimens were digitally photographed using a Panasonic HDD-H80 camera.

\section{RESULTS}

The sphenoid paranasal sinus is separated into two-sided cavity with a bony septum, but it is preferable to regard them at first as a single sinus. Its growth is nearly uniform, since the two-sided principal sinus cavity advances approximately to the correspond extension posteriorly.

In about $65.2 \%$ of cases, the lateral recess develops from the principal or main sinus cavity. This may extends to aerated anterior clinoid processes, or greater wings of sphenoid bone alongside the posterior wall of the orbit, with/without the pterygoid plates (Figure 1A \& B). This lateral recess is mostly asymmetrical on both sides; therefore, it must not be considered as a destructive lesion.
The sella turcica can be appear like a prominence within the sphenoid sinus roof and considered as one of the most important anatomical landmarks to the sellar floor. The degree or grade of sphenoid paranasal pneumatization differs greatly and its pattern significantly affects the sella, therefore a well-pneumatized sinus can disfigure its anatomical configurations. The criteria for recognition the conchal type or pattern is indicated by the existence of a solid bone under the sella lacking any visible pneumatization.

According to extent of pneumatization, sphenoid sinus can be categorized into three patterns or types:

1. The pre-sellar type that is characterized when the pneumatization is restricted to the area in front of the plane, which is in parallel with the anterior wall of sella turcica. In the present study, this type was the less common seen type with only $11.2 \%$ prevalence (Figure 2A).

2. The sellar type that is characterized when the extension of pneumatization is behind the anterior wall of sella turcica but it does not pierces the posterior wall of sella turcica; this type was found in $14 \%$ of cases (Figure 2B).

3.The post-sellar type that is characterized when the extension of pneumatization is behind the posterior sellar wall; this type was the most common type of pneumatization with $74.8 \%$ of cases (Figure 2C).
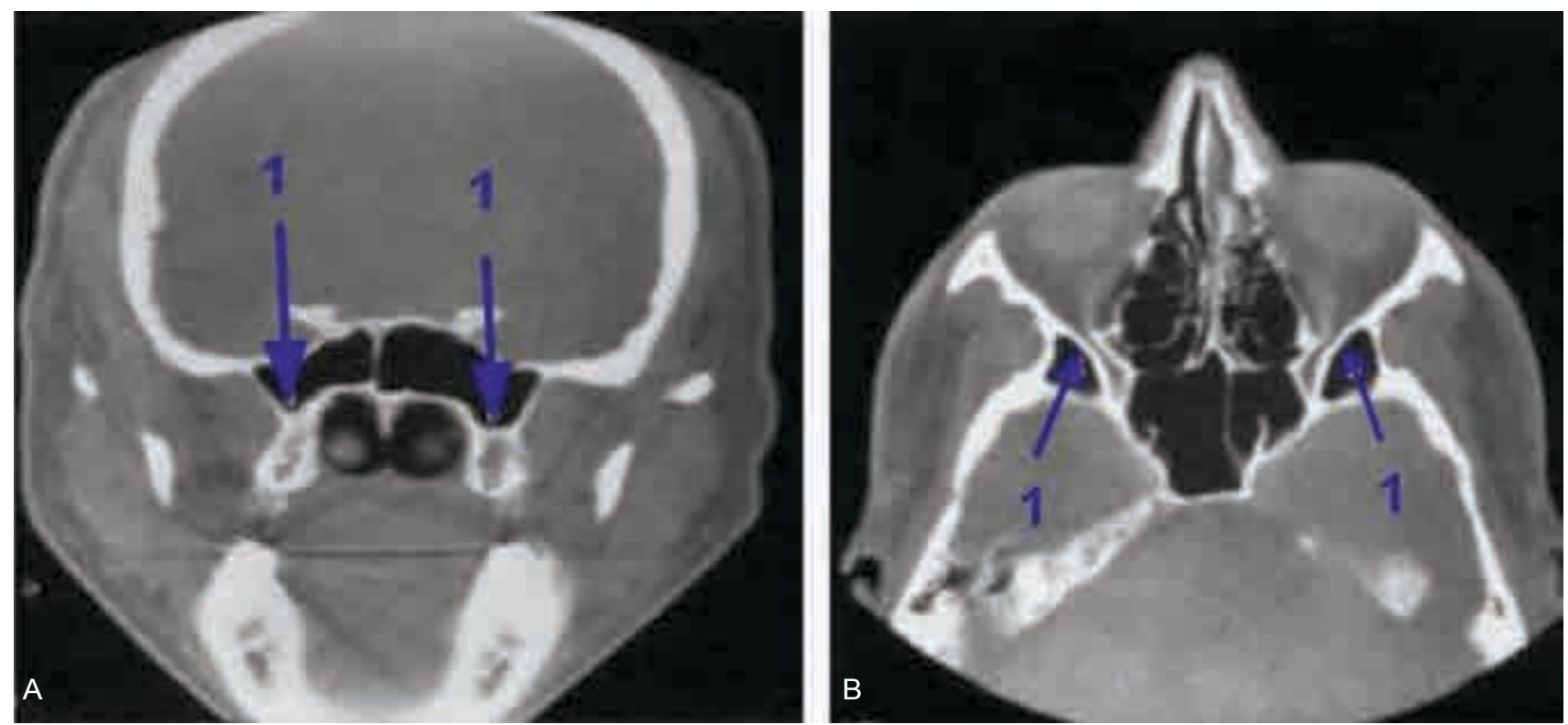

FIGURE 1. Pneumatization of the sphenoid sinus through the lateral recesses. $A$ - Coronal CT section showing $1^{*}$ its pneumatization down into the pterygoid plates. B - Axial CT section showing $1^{*}$ its pneumatization bilaterally into the posterior orbital wall. 


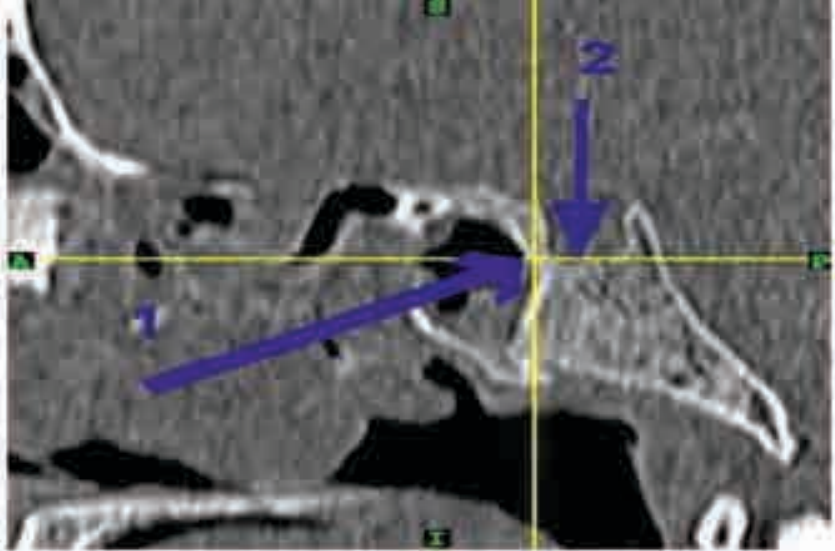

A

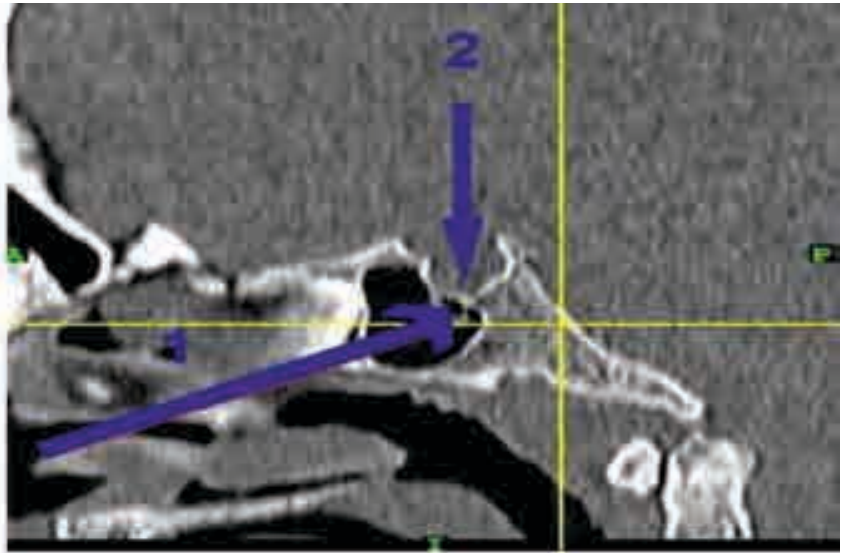

B

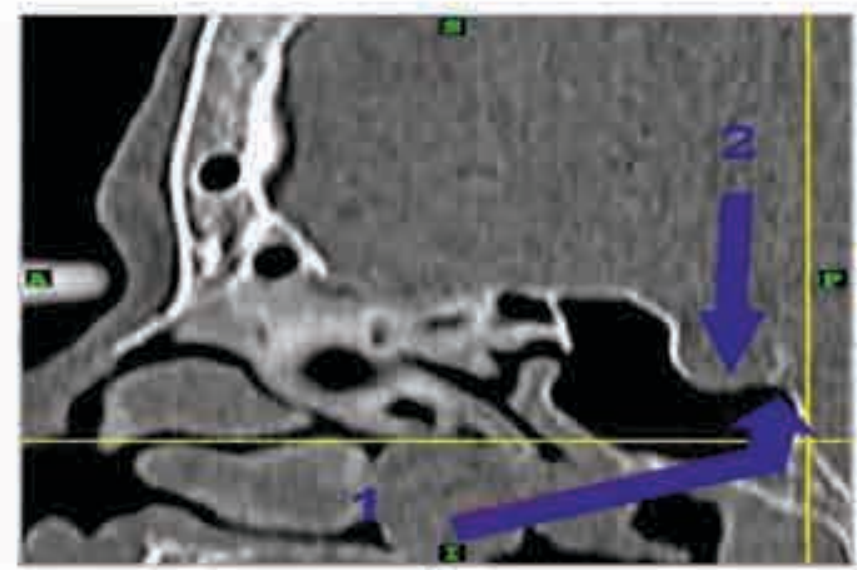

C

FIGURE 2. Sagittal CT sections showing the types or patterns of sphenoid sinus pneumatization. A - 1* The pre-sellar type; $2^{*}$ Sella turcica. $B-1^{*}$ The sellar type; ${ }^{*}$ Sella turcica. $C-1{ }^{*}$ The post-sellar type; $2^{*}$ Sella turcica.

All these types were best revealed in sagittal CT sections.

\section{Extent of pneumatization patterns of the in-} ternal carotid artery within the sphenoid sinus

It depended upon the degree of pneumatization of the sphenoid paranasal sinus around the retro-sellar part of internal carotid artery; therefore, in present study, the degree or grade of exposure inside the sinus was the basic role to demonstrate the following types:

1. Type 1 - Represents non-exposure because of the presence of a thick bony segment separating internal carotid artery from its adjacent sphenoid sinus. This type was found in 10\% of cases (Figure 3A).

2. Type $2-$ Represents exposure less than 90 degrees because of the presence of a thin bony segment separating internal carotid artery from its adjacent sphenoid sinus. The prevalence of this type was $30.8 \%$ cases (Figure 3B).

3. Type 3 - Represents exposure more than 90 but less than 180 degrees because the sphenoid si- nus lumen protrudes slightly by the internal carotid artery. This type was the most common type shown in $56 \%$ of cases.

4. Type 4 - Represents exposure more than 180 degrees because the sphenoid sinus lumen protrudes prominently by the internal carotid artery. This type was the less common type, since only $3.2 \%$ of cases were seen with this finding (Figure 3C).

All these four types were best shown in axial CT sections.

Extent of pneumatization patterns around optic nerve in the sphenoid sinus

In this study, the degree or grade of pneumatization into the neighborhood area of optic nerve can be classified into five different types:

1. Type 1 -Demonstrates no adjacent pneumatization, only $4 \%$ of cases were with this finding (Figure 4A).

2. Type 2 -Demonstrates pneumatization close to optic nerve without any indentation in sphenoid sinus. This type was the most common type of 


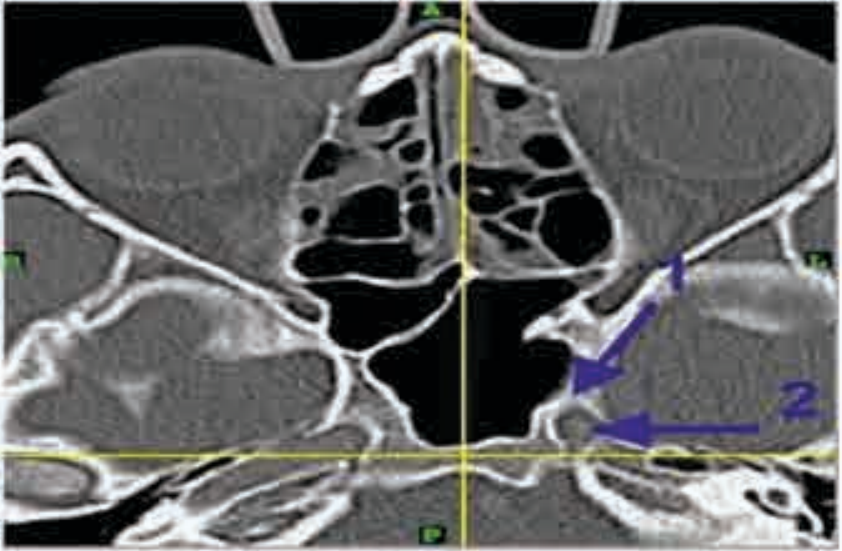

A

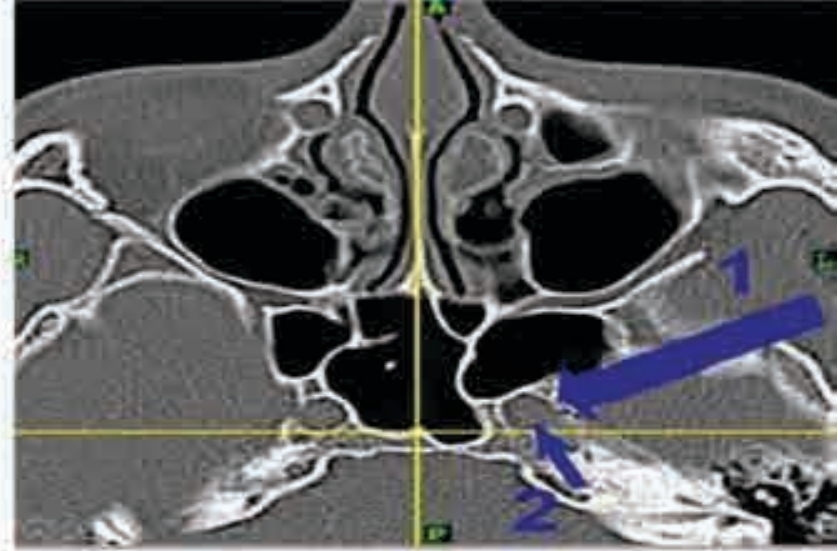

B

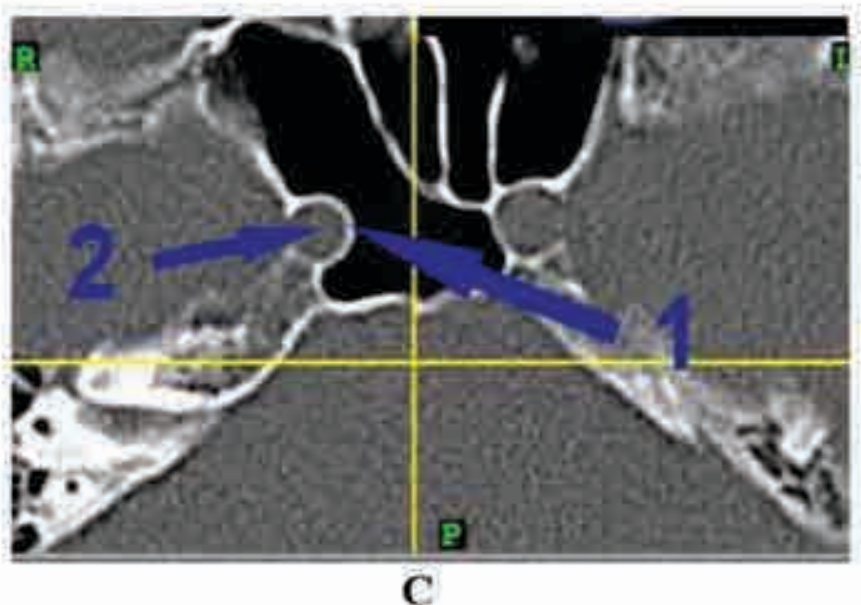

FIGURE 3. Axial CT sections showing different types of extent of sphenoid pneumatization around the retro-sellar segment of the internal carotid artery. $A-1^{*}$ Type $1 ; 2 *$ Internal carotid artery. $B-1$ * Type 2; 2* Internal carotid artery. $C-1{ }^{*}$ Type $4 ; 2 *$ Internal carotid artery.

pneumatization which was found in $52 \%$ of cases (Figure 4B).

3. Type 3 - Demonstrates pneumatization close to optic nerve associated with indentation in sphenoid sinus. The prevalence of this type was $34 \%$ of cases (Figure 4C).

4. Type 4-Demonstrates pneumatization of approximately less than fifty percentage of optic nerve circumference. This type was found in $3.6 \%$ of cases (Figure 4D).

5. Type 5 - Demonstrates pneumatization of further than fifty percentage of optic nerve circumference. This type was present in $6.4 \%$ of cases (Figure 4E).

All these five types were best seen in both coronal and axial CT sections.

Indeed, the anatomical position of internal carotid artery at the lower part of sphenoid paranasal sinus makes it less possible to get injury. Furthermore, it was obvious in this study, the size of internal carotid artery was greater than that of optic nerve; with a notable protection either by a thick bony segment, or when it close to sinus, acted to cause a bulge in internal carotid artery. In the present study, it was found that the 3,4 and 5 of optic nerve patterns of pneumatization corresponding to 2, 3 and 4 of internal carotid artery patterns of pneumatization.

\section{DISCUSSION}

The majority of sphenoid sinus was of post-sellar pattern or type of pneumatization with prevalence $74.8 \%$ of cases, which could be a result of the exclusion policy for those individuals who had sellar pathological lesions that might involve the sphenoid sinus. In Hamid et al. (20) study, pre-sellar, sellar and post-sellar types of pneumatization reported in $21 \%, 54.3 \%$, and $22.7 \%$ respectively; and the lateral extent of pneumatization into the greater wings of sphenoid bone that causing an obvious capacious sinus, was revealed with $15.8 \%$ of total cases. 


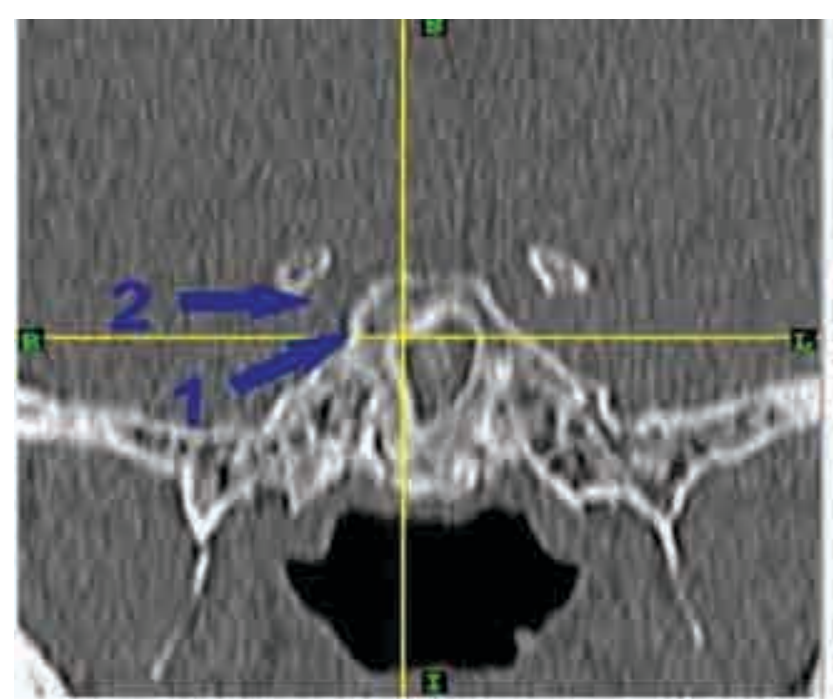

A

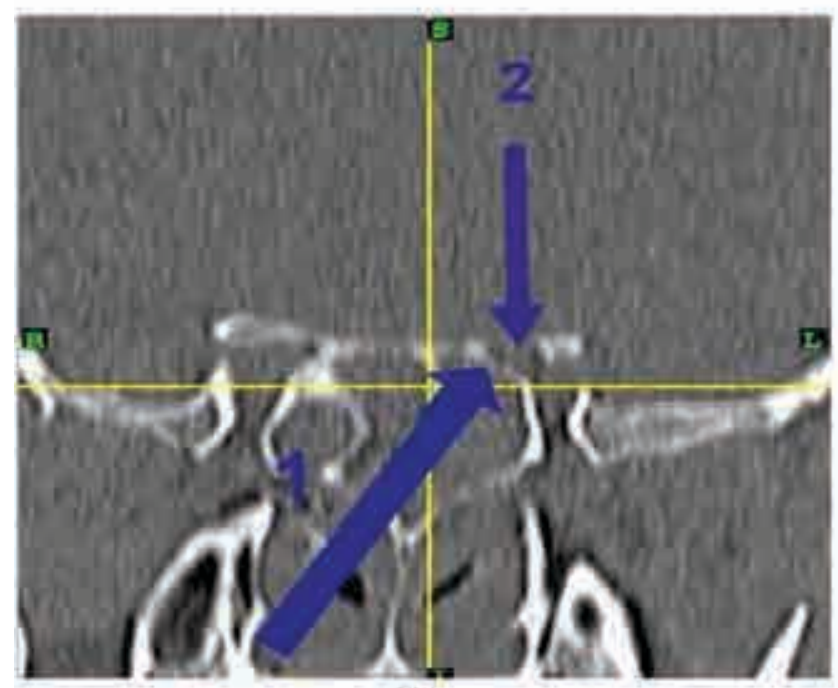

C

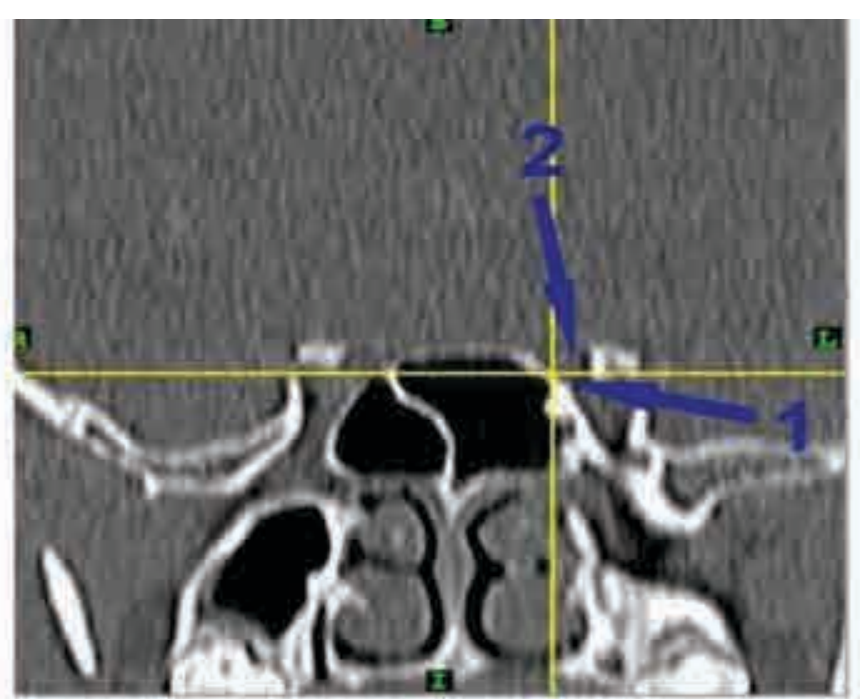

B

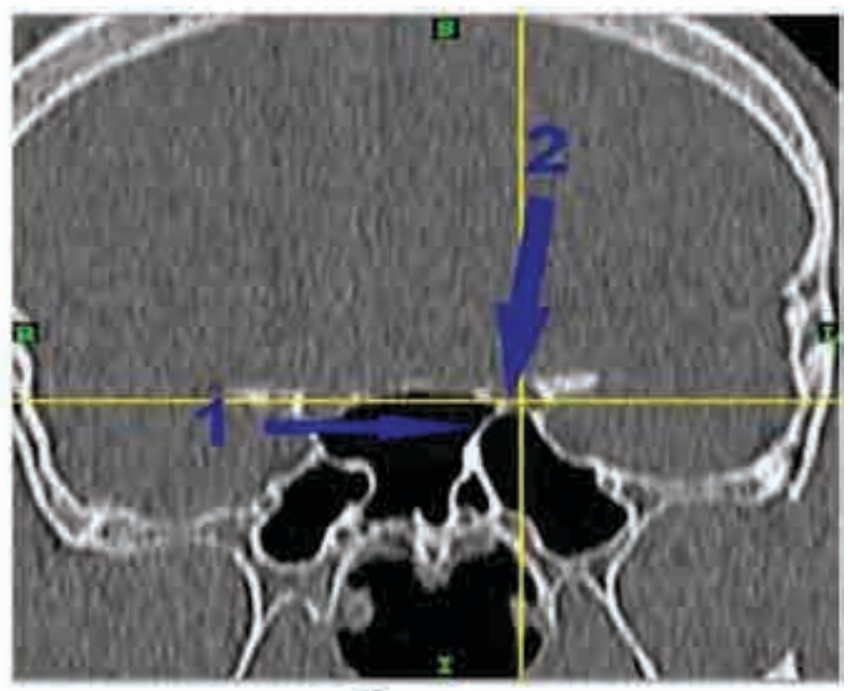

D

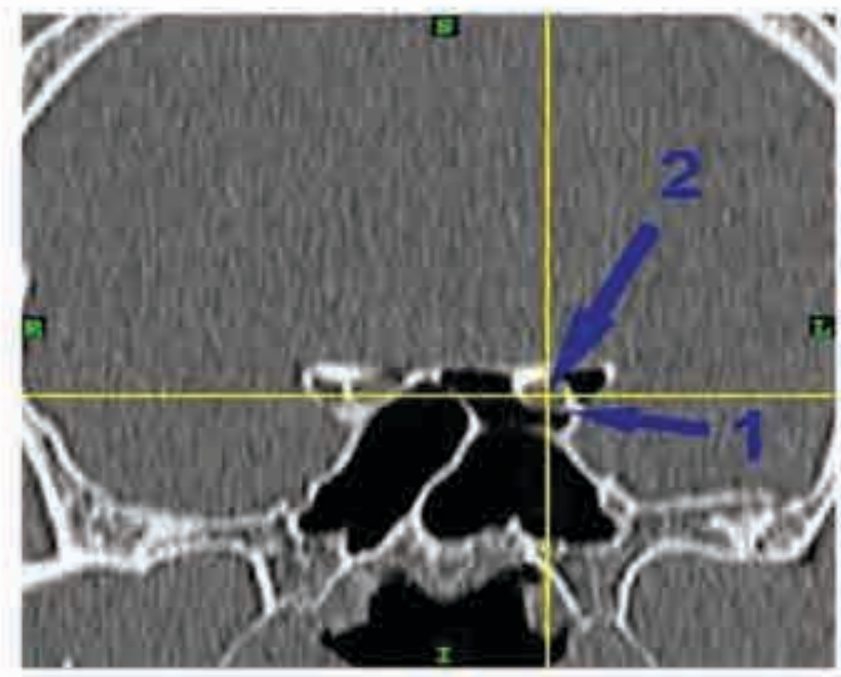

E

FIGURE 4. Coronal CT sections showing different types of extent of pneumatization for the optic nerve into the sphenoid sinus. $A-1^{*}$ Type $1 ; 2^{*}$ Optic nerve. $B-1^{*}$ Type $2 ; 2^{*}$ Optic nerve. $C-1^{*}$ Type $3 ; 2^{*}$ Optic nerve. $D-1^{*}$ Type 4; 2* Optic nerve. E-1* Type 5; 2* Optic nerve. 
The conchal type of sphenoid sinus were absent in this study, but was recorded in $2 \%$ of patients by Hamid et al. (20), whereas the rareness of conchal pneumatization agrees with other various researches done by Banna \& Olutola (21), Vaezi et al. (22) and Dal Secchi et al. (23).

The mature configuration of the sphenoid paranasal sinus was in significant relationship with internal carotid artery and the optic nerve, since these two structures were simply noticed as bulges at the lateral margin of this sinus. Septae inserting onto the internal carotid artery with optic nerve were found in $50 \%$ \& $8 \%$ respectively, compared to $37.5 \%$ \& $3 \%$ by Gibelli et al. (24) and $26.4 \%$ \& $4 \%$ by Alaa et al. (25) in the same order.

The present study allowed identification of three basic patterns of sphenoid sinus that explain the relation between the presence of an accessory septum and synchondrosis of different ossification centers, with eventual resistance in their junctions. Therefore, a hypothesis advanced by Som et al. (26) described the existence of an accessory septum may not completely enhanced, also in addition to their description of the relation between the septum and synchondrosis, they regarded those structures to be exist in every sinonasal complex. Actually, the present findings demonstrated a quite regular pattern related to the septal position at the posterior wall of sinus. The prevalence of septae in present study was $22 \%$ of cases, but if compared to studies, which described intersinus septae in about $68 \%$ of total cases, by Famurewa et al. (27). Those

\section{REFERENCES}

1. Berkovitz B \& Bernard JM. Head and neck anatomy a clinical reference. London: Martin Dunitz Ltd; 2002.

2. Hengerer AS. Embryonic development of the sinuses. Ear Nose Throat J. 1984;63:134.

3. Wani AH, Parry AH, Feroz I, Choh NA, Gojwari TA. Prevalence of different types of sphenoid sinus pneumatization in the Indian population: A noncontrast computed tomography-based study. Acta Med Int. 2019;6:74-7.

4. Cellina M, Gibelli D, Floridi C, Toluian T, Pittino CV, Martinenghi C, et al. Sphenoid sinuses: pneumatization and anatomical variantswhat the radiologist needs to know and report to avoid intraoperative complications. Surg Radiol Anat. 2020;42:1013-24.

5. Štoković N, Trkulja V, Dumić-Čule I, Čuković-Bagić I, Lauc T, Vukičević S, et al. Sphenoid sinus types, dimensions and relationship with surrounding structures. Ann Anat. 2016;203:69-76.

6. Movahhedian N, Paknahad M, Abbasinia F, Khojatepour L. Cone Beam Computed Tomography Analysis of Sphenoid Sinus Pneumatization and Relationship with Neurovascular Structures. J Maxillofac Oral Surg. 2020. recorded attachment of these septae into the clivus with $63.8 \%$ of cases reported by Elkammash et al. (28), while the closest study done by Idowu et al. (29) who was with only $27 \%$ of their studied cases.

\section{CONCLUSIONS}

This study observed the absence of the conchal pattern of sphenoid sinus pneumatization, with three obvious types of its pneumatization (the pre-sellar, sellar and post-sellar). In addition, the internal carotid artery was pneumatized within sphenoid sinus in four differentiated types with prevalence $10 \%, 30.8 \%, 56 \%$ and $3.2 \%$ respectively; while the optic nerve was pneumatized within the same sinus in five distinguishable types with prevalence $4 \%, 52 \%, 34 \%, 3.6 \%$ and $6.4 \%$ respectively had been reported. A higher prevalence of optic nerve extension into the lateral sphenoid sinus wall that causes bulging of optic canal in the same sinus. All these findings may suggest that superio-posterior margin of the sinus tends to show more pneumatization than other aspects. U1timately, these findings can be with magnificent importance to determine the gender, age or even race and ethnic group of alive or dead individuals.

\section{Acknowledgement}

The author grateful to all doctors and health workers at Radiology Section of both Baghdad Medical City and Salah Aldeen Teaching Hospitals for their magnificent assistance and unlimited support in order to carry out this research successfully.

Conflict of interest: none declared Financial support: none declared

7. Mohammad SA, Abdalla MA, Mahdi AJJ. Orbitometry of orbital opening and orbital cavity in neonate compared adult. Tik Med J. 2011;210-6.

8. Rahmati A, Ghafari R, AnjomShoa M. Normal variations of sphenoid sinus and the adjacent structures detected in cone beam computed tomography. J Dent (Shiraz). 2016;17:32-37.

9. Wiebracht ND, Zimmer LA. Complex anatomy of the sphenoid sinus: A radiographic study and literature review. J Neurol Surg B Skull Base. 2014;75:378-82.

10. Aksoy F, Yenigun A, Goktas SS, Ozturan O. Association of accessory sphenoid septa with variations in neighboring structures. J Laryngol Otol. 2017;131(01):51-5.

11. Farhan N, Naqvi SU, Rasheed B, Sattar A, Khan M, Rahim A, et al. Identification of Significant Anatomical Variations in the Nose and Anterior Skull Base Using Computed Tomography: A CrossSectional Study. Cureus. 2020;12(6):e8449.

12. Cappella A, Gibelli D, Cellina M, Mazzarelli D, Oliva AG, De Angelis $D$. Three-dimensional analysis of sphenoid sinus uniqueness for assessing personal identification: a novel method based on 3D-3D superimposition. Int J Legal Med. 2019;133(6):1895-901. 
13. Alshaikh $\mathrm{N}$, Aldhurais A. Anatomic variations of the nose and paranasal sinuses in Saudi population: computed tomography scan analysis. Egypt J Otolaryngol. 2018;34:234-41.

14. Abdalla MA, Mahdi AJJ. Maxillary Sinus Measurements in Different Age Groups of Human Cadavers. Tik J Dent Sci. 2013;1:107-12.

15. Ramalho CO, Marenco HA, de Assis Vaz, Guimarães Filho F, da Costa MD, et al. Intrasphenoid septations inserted into the internal carotid arteries: A frequent and risky relationship in transsphenoidal surgeries. Braz J Otorhinolaryngol. 2017;83:162-7.

16. Souadih K, Belaid A, Ben Salem D, Conze PH. Automatic forensic identification using 3D sphenoid sinus segmentation and deep characterization. Med Biol Eng Comput. 2020;58,291-306.

17. Cohen O, Warman M, Fried M, Shoffel-Havakuk H, Adi M, Halperin $\mathrm{D}$, et al. Volumetric analysis of the maxillary, sphenoid and frontal sinuses: a comparative computerized tomography based study. Auris Nasus Larynx. 2018;45(1):96-102.

18. Gibelli D, Cellina M, Gibelli S, Oliva AG, Termine G, Sforza C. Anatomical variants of sphenoid sinuses pneumatization: a CT scan study on a Northern Italian population. Radiol Med. 2017;122(8):575-80.

19. Balegh $\mathrm{AH}$, Moustafa T. Anatomical Variations of Paranasal Sinuses on Multidetector Computed Tomography. On J Otolaryngol \& Rhinol. 2020;3(2):556

20. Hamid O, El Fiky L, Hassan O, Kotb A \& El Fiky S. Anatomic variations of the sphenoid sinus and their impact on trans-sphenoid pituitary surgery. J Neurosurg. 2008;18(1):9-15.

21. Banna M, Olutola PS. Patterns of pneumatization and septation of the sphenoidal sinus. J Can Assoc Radiol. 1983;34(4):291-3.

22. Vaezi A, Cardenas E, Pinheiro-Neto C, Paluzzi A, Branstetter BF, Gardner PA, et al. Classification of sphenoid sinus pneumatization: relevance for endoscopic skull base surgery. Laryngoscope. 2015;125(03):577-81.

23. Dal Secchi MM, Dolci RL, Teixeira R, Lazarini PR. An analysis of anatomic variations of the sphenoid sinus and its relationship to the internal carotid artery. Int Arch Otorhinolaryngol. 2018;22:161-6.

24. Gibelli D, Cellina M, Gibelli S, Cappella A, Oliva AG, Termine G. Relationship between sphenoid sinus volume and protrusion of internal carotid artery and optic nerve: a 3D segmentation study on maxillofacial CT-scans. Surg Radiol Anat. 2019;41(5):507-12.

25. Alaa A, Mahdy M, Ghonemi N. The sensitivity of optic nerve computed tomography scan vs optical coherence tomography in diagnosis of Idiopathic intracranial hypertension. Zag Uni Med J. 2020;26(1):108-13.

26. Som PM, Lawson W, Fatterparker GM. Embryology, anatomy, physiology and imaging of the sinonasal cavities. In: Som PM, Curtin HD (eds). Head and neck imaging, 5th ed. Elsevier Health Sciences, St Louis; 2011.

27. Famurewa OC, Ibitoye BO, Ameye SA, Asaleye CM, Ayoola OO, Onigbinde OS. Sphenoid sinus pneumatization, septation, and the internal carotid artery: A computed tomography study. Niger Med J. 2018;59:7-13.

28. Elkammash TH, Enaba MM, Awadalla AM. Variability in sphenoid sinus pneumatization and its impact upon reduction of complications following sellar region surgeries. Egypt J Radiol Nucl Med. 2014;45:705-14.

29. Idowu OE, Balogun BO, Okoli CA. Dimensions, septation, and pattern of pneumatization of the sphenoidal sinus. Folia Morphol (Warsz). 2009;68:228-32. 